\title{
On transport in porous formations characterized by heterogeneity of evolving scales
}

\author{
Alberto Bellin, ${ }^{1}$ Marilena Pannone, ${ }^{2,3}$ Aldo Fiori, ${ }^{4}$ and Andrea Rinaldo ${ }^{2}$
}

\begin{abstract}
Solute transport in natural formations at the regional scale is influenced by several scales of heterogeneity which correspond to the presence of several geological units called facies. As customarily assumed in stochastic theories, inside the facies the transport can be characterized by a single scale of heterogeneity. At the regional scale several geological units are present such that a hierarchy of relevant scales needs to be defined. A possible model for this spatial variability assumes the log conductivity as a random space function of stationary increments characterized by a power law semivariogram. With this hypothesis the ergodic dispersion coefficient grows unbounded as time increases, leading to the phenomenon called anomalous dispersion. An alternative approach considers the plume in nonergodic conditions and assumes the effective dispersion coefficient, which is defined through differentiation in time of the expected value of the spatial second-order plume moment, as representative of macrodispersion. Large differences have been observed in the resulting plume spreading while approaching the problem using the above alternative definitions. In this paper we provide first-order analytical solutions for the longitudinal effective dispersion coefficient, $D_{L}$, as well as for the expected value of the longitudinal spatial plume moment, $\left\langle S_{11}\right\rangle$, that complement semianalytical expressions recently proposed in literature. Furthermore, we provide a semianalytical expression for the standard deviation of the longitudinal second-order moment which is important in assessing the interval of confidence of the estimation provided by $\left\langle S_{11}\right\rangle$. Suitable numerical simulations are performed to validate analytical and semianalytical expressions as well as to assess the impact of the cutoff in the log conductivity power spectrum imposed by choosing a finite domain dimension. We conclude that according to recently published results, the dispersion is anomalous when the Hurst coefficients, $H$, is larger than 0.5 while it is Fickian for $H<0.5$. This is in contrast with the ergodic analysis which concludes that the dispersion is anomalous irrespective of the Hurst coefficient. Hence the effective dispersion coefficient is more effective than the ergodic dispersion coefficient to represent the plume spreading.

However, the standard deviation of the longitudinal spatial second-order moment is of the same order of magnitude as the expected value leading to the conclusion that the estimations provided by $D_{L}$ and $\left\langle S_{11}\right\rangle$ are affected by large uncertainties. Numerical results are in good agreement with the analytical solutions, and under some hypotheses they are not influenced by the cutoff. This is not the case for the ergodic second-order longitudinal moment, which strongly depends on the imposed cutoff.
\end{abstract}

\section{Introduction}

Modeling the strong spatial variability exhibited by natural formations like gas or oil reservoirs and aquifers is a crucial task when transport processes are addressed. Most stochastic theories [see, e.g., Dagan, 1989] assume that the log conductivity $Y(\mathbf{x})=\ln K(\mathbf{x})$, where $K$ is the local hydraulic conductivity of the formation and $\mathrm{x}$ is the vector of spatial coordinates,

\footnotetext{
${ }^{1}$ Dipartimento di Ingegneria Civile e Ambientale, Università di Trento, Trento, Italy.

${ }^{2}$ Istituto di Idraulica "G. Poleni," Università di Padova, Padova, Italy.

${ }^{3}$ Also at Dipartimento di Ingegneria e Fisica dell'Ambiente, Università della Basilicata, Potenza, Italy.

${ }^{4}$ Dipartimento di Scienze dell'Ingegneria Civile, Terza Università di Roma, Rome, Italy.

Copyright 1996 by the American Geophysical Union.

Paper number 96WR02507.

0043-1397/96/96WR-02507\$09.00
}

is a stationary random space function (RSF), normally distributed and thus fully characterized by the constant mean $\langle Y\rangle$ and the isotropic exponential covariance function $C_{Y}(r)=\sigma_{Y}^{2}$ $\exp \left[-r / I_{Y}\right]$ (with $r=|\mathbf{r}|$, where $\mathbf{r}$ is the two-point isotropic lag, $\sigma_{Y}^{2}$ is the variance, and $I_{Y}$ is the integral scale). Embedded in such assumption is the existence of a fundamental characteristic scale of heterogeneity, that is, the integral scale $I_{Y}$. Nevertheless, natural formations are generated by processes that create distributions of geologically homogeneous units (facies) and, according to Neuman [1990], heterogeneities resulting from the intertwined arrangement of facies are characterized by a complex hierarchy of scales.

Whether such hierarchy is an infinite one is difficult to assess experimentally, but it is reasonable to argue, along with $\mathrm{Neu}$ man [1990], that as the lag increases, larger scales come into play, causing the semivariogram of $Y$ to increase in some stepwise manner. To describe such media, Neuman [1990] adopted the simple scaling assumption, yielding the following semivariogram: 


$$
\gamma_{Y}(r)=\frac{1}{2}\left\langle[Y(\mathbf{x}+\mathbf{r})-Y(\mathbf{x})]^{2}\right\rangle=a(r / I)^{\beta} \quad 0<\beta .2
$$

where $I$ is a suitable reference scale, $a$ is a constant, and the scaling exponent $H=\beta / 2$ is called the Hurst coefficient [e.g., Feder, 1988]. Notice that for $0<H \leq 1$ variance and integral scale of the field $Y$ grow infinitely, thus describing a medium with no preferential scale. This singular behavior has been popularized by Mandelbrot [1983] through the "syndrome of infinite variance." Notice also that the semivariogram given by (1) shows a continuous growth with distance, somewhat different from what we expect in nature. The presence of more than one reference scale of variability has an influence on transport. Recently, Rubin [1995] showed that for formations with two characteristic scales, as for example the sand-shale or sandy clay formations, macrodispersion grows with a rate that depends on the ratio between the scales.

The discussion on the generality of the scale-free model (1) has been (and still is) lively. Anderson [1991] pointed out that the model (1) has a geologic limit (i.e., an embedded scale) determined by the areal extent of the geologic environment, while Neuman [1991, 1994] claims that it models well experimental data. The hypothesis that the spatial variability of the hydraulic conductivity is represented by a continuous hierarchy of scales has been recently addressed by several authors [Pickens and Grisak, 1981; Gelhar, 1986; Sposito et al., 1986; Lake, 1989; Neuman, 1990, 1994; Kemblowski and Wen, 1993; Dagan, 1994; Desbarats and Bachu, 1994; Rajaram and Gelhar, 1995]. One issue concerns stationarity. It is commonly accepted that although at the regional scale complex geologic systems are hardly stationary, in relatively homogeneous sites, such as Borden [Sudicky, 1986] or Cape Cod [Hess et al., 1992], the stationarity assumption may be appropriate.

The presence of significant trends or of a preponderance of heterogeneity scales characterizes aquifers to a degree that depends on the complexity of the geologic formation. Nonstationarity has been detected at the Columbus site, which is characterized by a field scale comparable with that of Borden and Cape Cod but with a much more complex geological structure [Boggs et al., 1992]. At the regional scale the formation horizontal dimension is of the order of tens to hundreds of aquifer thicknesses, such that the appropriate flow and transport models are essentially two-dimensional. In such a case, aquifer heterogeneities, described by the hydraulic log transmissivity $Y\left(x_{1}, x_{2}\right)=\ln T\left(x_{1}, x_{2}\right)$, are clearly influenced by the depositional process. For example, the deposits forming an outwash experience sorting [Anderson, 1991].

Although at the regional scale the existence of several scales of heterogeneity seems reasonable, clear direct experimental evidence cannot be gathered to support model (1). Using regression analysis, Neuman [1990] provided an indirect justification of (1), inferred from the scaling behavior observed for the longitudinal dispersivity of tracer plumes. It should be noted that the observational foundations as well as the implications of the scaling assumption have been extensively debated [Gelhar et al., 1992; Neuman, 1993; Gelhar et al., 1993; Dagan, 1994].

Although there is growing evidence that the log conductivity field of porous and fractured geologic media show self-affine properties [Neuman, 1995], the proper representation of the natural heterogeneity of the aquifers is still an open question mainly because observations covering a suitably large number of spatial scales are still lacking. In this context (1) represents the simplest choice among models showing a scaling behavior. A possible limitation of the model adopted is then the simple scaling assumption. Although interwinded sets of scaling exponents possibly offer a reasonable model for the complexity observed in nature, no experimental evidence is available to date to support such an assumption. In this paper, we adopt, following Dagan [1994], the simple scaling assumption which is suggested to be effective in reproducing the features of some natural formations.

As customary in transport studies [Dagan, 1989], the spreading is characterized by the definition of a suitable dispersion coefficient. Limiting the attention to the longitudinal direction, that is, the direction of the mean flow which is indicated with the subscript $L$ (or 1), two different dispersion coefficients can be defined: the ergodic dispersion coefficient $d_{L}$ and the effective dispersion coefficient $D_{L}$. The differences between them had been described by Fisher et al. [1979] in the context of turbulent mixing and by Kitanidis [1988], Dagan [1991], and Rajaram and Gelhar [1993] in the context of transport in natural single-scale porous formations. For evolving scale formations, Glimm et al. [1993] discussed the interplay of geologic and plume scales, concluding that heterogeneities present at scales larger than that of plume size lead to an increased dispersion coefficient with respect to the case in which they are considered deterministic. This conclusion is consistent with Dagan's [1991] findings that the ergodic dispersion coefficient $d_{L}$ is constituted by two additive components: (1) the effective dispersion coefficient $D_{L}$, related to the heterogeneities at the plume scale, and (2) the dispersive effect due to the uncertainty in the plume centroid localization, which is related to heterogeneities at scales larger than the plume size. The implications of the ergodic assumption on transport in evolving scale formations has been discussed by Dagan [1994].

A comprehensive theoretical analysis of the scaling properties of the ergodic longitudinal dispersion coefficient has been conducted in a series of papers [Glimm and Sharp, 1991; Furtado et al., 1990, 1991; Zhang, 1992]. In these works a log conductivity covariance function has been assumed in the power law form: $C_{Y}(r)=a r^{\beta}$. The main conclusions of the above works are the following [Glimm et al., 1993]: (1) for $\beta<$ $-1, C_{Y}$ is rapidly decreasing to zero and the transport is Fickian; (2) for $-1 \leq \beta \leq 0, C_{Y}$ is slowly decreasing and is characterized by an unbounded integral scale such that $d_{L}$ scales as $d_{L} \sim a U L^{\gamma}$. Perturbation theory and numerical solutions show that for large travel distances, the exponent $\gamma$ varies in time approaching a constant value [Glimm et al., 1992]. Finally, for $\beta>1$ the asymptotic behavior of $d_{L}$ depends on the cutoff, that is, the finite size of the field. According to the above ergodic results (refer to Glimm et al. [1993] for a review), the transport is Fickian for rapidly decreasing correlations and anomalous for slowly decreasing (or increasing) correlations.

This paper hinges on the recent work of Dagan [1994], who obtained exact first-order solutions for the effective dispersion coefficients for formations described by model (1). The main result of the paper is that the so-called ergodic assumption, implicit in most theoretical results of stochastic models, cannot be assumed as generally valid. As a result, rather than showing an anomalous continuous growth, the effective dispersion reaches a Fickian asymptotic limit for $\beta<1$. This is in marked contrast with previous theoretical results. The anomalous case 
is recovered for $\beta>1$, but stationarity issues become important.

In this paper we present a few novel first-order exact solutions for the effective dispersion coefficient and for the mean and variance of the longitudinal second-order moments of a dispersing plume. Our solutions for the effective dispersion complements that of Dagan [1994] by relaxing some simplifying assumptions. The analytical solutions are then compared with suitable numerical solutions of the transport equations in a nonlinear Monte Carlo framework.

The paper outline is as follows: the next section presents the theoretical background and our novel exact solutions. Section 3 discusses the numerical simulations performed in order to explore the inference of the linearity assumptions, both in the flow and the transport equations, embedded into the exact solutions. A discussion and a set of conclusions then close the paper.

\section{Theoretical Background and Novel Analytical Results}

The starting point of this paper is the work by Dagan [1994] on the significance of evolving scale heterogeneities in solute transport. The hydraulic $\log$ transmissivity $Y=\ln T$ is assumed stationary in the increments with semivariogram defined by (1), and pore-scale dispersion is neglected. The last hypothesis is justified at least for isotropic formations and values of local dispersivity encountered in practice [Fiori, 1996]. The key results of Dagan [1994] are briefly recalled. The spreading of plumes is described by focusing on longitudinal dispersion coefficients.

The ergodic coefficient $d_{L}$ implies that in each realization of the random process the centroid of the dispersing plume can be reasonably well approximated by the mean trajectory, say, $\left\langle R_{1}(t)\right\rangle$ (which, in turn, is the ensemble mean trajectory of the single particle). This postulates that $R_{11}(t) \sim 0$, where $R_{11}(t)$ is the second-order moment of the cloud made up by the points labeling the plume centroid in independent realizations. The classic definition of the dispersive process is then given [e.g., Fisher et al., 1979; Dagan, 1984, 1989] by the relationship

$$
d_{L}(t)=\frac{1}{2} \frac{d X_{11}(t)}{d t}
$$

where $X_{11}(t)$ is the second-order moment of the residual particle displacements about the mean trajectory.

Nonergodic plumes experience wide fluctuations of the actual trajectories $R_{1}(t)$ resulting in a non-null value of $R_{11}(t)$. For ergodic plumes $d_{L}=D_{L}$, that is, $R_{1}(t) \sim\left\langle R_{1}(t)\right\rangle$ and $R_{11}(t) \sim 0$, while for nonergodic plumes the following equation holds [Dagan, 1991]:

$$
D_{L}(t)=d_{L}(t)-\frac{1}{2} \frac{d}{d t} R_{11}(t)
$$

Hence $R_{11}$ is related to the uncertainty in the localization of the actual plume centroid. Equation (3) is derived [Dagan, 1994] from the dual relationship involving the second-order longitudinal plume moment [Dagan, 1991]:

$$
\left\langle S_{11}(t)\right\rangle=S_{11}(0)+X_{11}(t)-R_{11}(t)
$$

where $\left\langle S_{11}(t)\right\rangle$ is the expected value of the actual longitudinal second-order plume moment $\left(S_{11}(0)\right.$ is its initial value).

The conceptual and practical differences between $d_{L}$ and
$D_{L}$ can be highlighted by the following relationships (5) and (6). In fact one has

$$
\begin{aligned}
d_{L}(t) & =\frac{1}{2} \frac{d}{d t} \int_{\Omega}\langle C(\mathbf{x}, t)\rangle\left[x_{1}-\left\langle R_{1}(t)\right\rangle\right]^{2} d \mathbf{x} \\
& \sim \int_{0}^{t} u_{11}\left(\left\langle R_{1}(\tau)\right\rangle\right) d \tau
\end{aligned}
$$

where $u_{11}$ is the longitudinal velocity covariance function, $C$ is the solute concentration at time $t$ and position $\mathrm{x}, R_{1}(t)$ is the trajectory of the plume centroid, and $\Omega$ is the domain volume. Notice that (5) is a spinoff of Taylor's [1921] equivalence of ensemble mean concentrations and displacement probability distributions. In (5), $d_{L}$ results as a first-order approximation obtained by the Lagrangian approach replacing the actual particle trajectory with its expected value [Dagan, 1984]. In nonergodic conditions one has instead

$$
D_{L}(t)=\frac{1}{2} \frac{d}{d t}\left\langle\int_{\Omega} C(\mathbf{x}, t)\left[x_{1}-R_{1}(t)\right]^{2} d \mathbf{x}\right\rangle
$$

The main result embodied in (6), (3), and (4) is that for nonergodic plumes, the best estimate for the actual plume dispersion is $D_{L}$, while $d_{L}$ is affected by uncertainty in the plume centroid localization [Kitanidis, 1988; Dagan, 1991].

We have computed exact solutions for $D_{L},\left\langle S_{11}\right\rangle$ and $\operatorname{Var}\left[S_{11}\right]$ for the case described by the model equation (1) for the spatial variability of physical heterogeneity. Our solutions are presented in the appendix. Our results complement those given by Dagan [1994] in the following aspects: (1) We supplied an analytical solution to the quadratures employed in Dagan's [1994] derivation of $D_{L} ;(2)$ new exact solutions for $\left\langle S_{11}\right\rangle$ and the variance $\operatorname{Var}\left[S_{11}\right]$ are obtained; and (3) we have performed accurate numerical simulations to assess the range of applicability of the analytical solutions. The comparison between analytical and numerical solutions is performed with reference to $\left\langle S_{11}\right\rangle$ and $\operatorname{Var}\left[S_{11}\right]$ because the computation of longitudinal dispersion, $D_{L}$, by numerical differentiation of the actual longitudinal second-order moment $S_{11}$ is error prone.

Figures 5a-5c show the dimensionless expected value of $S_{11}$, $\left\langle S_{11}(t)\right\rangle$; its standard deviation, $\mathrm{SD}\left[S_{11}\right]$; and the coefficient of variation, $\mathrm{CV}\left[S_{11}\right]=\left\{\operatorname{Var}\left[S_{11}\right]\right\}^{1 / 2} /\left\langle S_{11}\right\rangle$ computed respectively through (A4), (A13), and (A14) and numerically for $\beta=0.75$ and $a=0.1$ in (1). The same quantities for $\beta=1.75$ and $a=0.0025$ are shown in Figures 6a-6c.

We observe that for $\beta \geq 1,\left\langle S_{11}\right\rangle$ grows faster than the mean dimensionless travel distance, leading to a rapid increasing of the longitudinal second-order moment with the travel distance. The coefficient of variation converges rapidly to the asymptotic value which depends solely on the value of $\beta$ and is close to $1 \mathrm{in}$ all cases. The practical result is that the interval of confidence, that is, the range of variability of the difference between the actual second-order moment $S_{11}$ and the estimate provided by $\left\langle S_{11}\right\rangle$, is of the same order of magnitude of $\left\langle S_{11}\right\rangle$ at all times.

Equations (A7) and (A8) confirm the asymptotic behaviors obtained by Dagan [1994] expanding $\gamma_{u}$ in a Taylor series truncated at the second order along the transverse direction, $x_{2}$, and about the point $\left(x_{1}, 0\right)$. The maximum growth rate of $D_{L}$ is then proportional to the mean dimensionless travel dis- 
tance leading to the result that $D_{L}$ grows with a power between 0 and 1 for $0<\beta \leq 2$. This agrees with the results obtained by Dagan [1994]. Thus, while the ergodic dispersion coefficient $d_{L}$ turns out to be unbounded irrespective of the value of $\beta$, the effective dispersion coefficient presents two cases: (1) For $0 \leq$ $\beta<1$ the transport is Fickian; the asymptotic value of the dispersion coefficient depends on $\beta$, that is, it grows as $l^{1+\beta}$, where $l$ is the transverse initial dimension of the plume. (2) For $1 \leq \beta \leq 2$ the dispersive transport is anomalous, that is, the dispersion coefficient grows unbounded; the dispersion coefficient grows as $\ln x$ for $\beta=1$ and as $x$ for $\beta=2$, leading to the results that for $0 \leq \beta \leq 2$ the maximum growing rate for large travel distances is linear.

Our results thus confirm and slightly extend the findings of Dagan [1994]. The new semianalytical solution for $\operatorname{SD}\left[S_{11}\right]$ is discussed in section 4 . The inference of the assumptions embedded into the analytic solution, chiefly the validity of the linearizations built into the flow and transport equations, remains to be seen. This will be addressed in the ensuing sections of this paper.

\section{Numerical Analysis}

In order to analyze in a nonlinear framework the results of section 2, we set up a numerical experiment in which the linearity hypotheses employed in the exact analysis of flow and transport were relaxed. An important issue in solving the problem numerically is the definition of a reference scale to render distances suitably dimensionless. Dagan [1994] used the transverse initial plume dimension $l$ as a reference scale for his analytical first-order solutions. This choice is effective for the analytical approach but not useful for the numerical solution because in such a case changes in the transverse initial plume dimension would also change the dimensionless field dimension and then also the infrared cutoff of the log conductivity power spectrum. Hence, to facilitate the numerical approach and to ensure the same field dimensions in each simulation, we relate the reference scale $I$ to the smaller scale that needs to be numerically resolved [Glimm et al., 1993] to make flow and transport insensitive to further grid refinement. The early suggestion of Ababou et al. [1989] relates the density of the computational lattice to the variance of the log conductivity. In this case we rely on the specific indications contained in the recent work by Bellin et al. [1992], suggesting that transport is not influenced by refinements of the grid size involving more than four points per reference scale. Therefore we choose the following numerical grid size: $\Delta x=I / 4$.

According to model (1), $\sigma_{Y}^{2}$ scales as

$$
\sigma_{Y}^{2} \propto L^{\beta}
$$

where $L$ is the characteristic scale of the flow domain. Hence the log-conductivity integral scale $I_{Y}$ shows the following scaling property:

$$
I_{Y} \propto \alpha L
$$

where $\alpha$ is a constant that depends on $\beta$. To assess the impact of boundary conditions on transport, we performed the numerical simulations considering different domain dimensions for a fixed longitudinal dimension $L_{1}=40 I$ by assuming the following values for the transverse one: $L_{2}=20 I$ and $40 I$.

The numerical simulations are organized as in the paper by Bellin et al. [1992] and made up by the following steps:
1. A normally distributed $\log$ transmissivity field $Y\left(x_{1}, x_{2}\right)$ of stationary increments and isotropic semivariogram $\gamma_{Y}(r)=$ $a(r / I)^{\beta}$ is generated over the domain; the transmissivity field is obtained by the transformation $T\left(x_{1}, x_{2}\right)=\exp \left[Y\left(x_{1}, x_{2}\right)\right]$.

2. The fully nonlinear flow equation is solved by the Galerkin finite element approach employing three nodes triangular elements with linear weight functions. The optimal mesh arrangement, that is, a scheme that satisfies the positive transmissivity condition, has been employed [Forsyth, 1991; Cordes and Putti, 1996]. The characteristic dimension of the elements is $0.25 I$. Local and global mass balance have been verified according to the method presented by Bellin et al. [1992, 1994]. The boundary conditions are impervious boundaries along the longer sides and imposed head at the other sides. The average and the standard deviation of the mean velocity computed for the case $\beta=0.75$ and with reference to 1500 Monte Carlo realizations are equal to, respectively, 1 and $10^{-7}$, leading to the conclusion that the mean velocity can indeed be assumed as constant and unit in each realization.

3. The transport is solved in the Lagrangian framework using a particle tracking approach and neglecting pore-scale dispersion as in the analysis of section 2 . The particles are injected along a strip of length $l$ normal to the mean flow direction with a density of 10 particles per reference scale $I$. The $i$ th particle is tracked according to the following expression: $\mathbf{X}^{i}(t)=\mathbf{X}^{t}(t-\Delta t)+\mathbf{v}\left(\mathbf{X}^{i}(t-\Delta t)\right) \Delta t$, where $\mathbf{X}^{t}(t)$ is the particle trajectory at time $t, \mathbf{v}(\mathbf{x})$ is the Eulerian velocity at position $\mathrm{x}$, and $\Delta t$ is the time step. Furthermore, particles are assumed noninteracting.

4. Steps 1-3 are repeated in a suitable [Bellin et al., 1992] Monte Carlo manner to compute expected values and variances of the plume spatial moments.

The generation of independent random $Y$ fields of stationary increments is a critical step in approaching the problem numerically. The generation is performed using the method recently developed by Bellin and Rubin [1996]. The method generates the field using a geostatistical approach that does not require the preventive definition of an "infrared cutoff," that is, the filtering of the wavelengths larger than the field dimension, from the $Y$ spectrum. The larger scale of variability is, however, limited by the field dimension, and the cutoff is automatically introduced by the fact that the generation is performed in a finite domain. The mean value of $Y$ is maintained constant in each realization. Since $Y$ is not stationary, the above constriction is statistically equivalent to the geostatistical conditioning of the mean velocity field to a given constant value.

Figures $1 \mathrm{a}$ and $1 \mathrm{~b}$ show an example of a log conductivity field with $a=0.1, \beta=0.75$ and the resulting velocity field, respectively. The reconstructed expected value of the log conductivity semivariogram and its standard deviation $\operatorname{SD}\left[\gamma_{Y}\right]$ are shown in Figures $2 a$ and $2 b$, respectively. The expected value and the standard deviation are computed in a Monte Carlo manner averaging over a number $M$ of independent realizations according to the following convergence criteria [Bellin and Rubin, 1996]:

$$
\left\{\frac{1}{N L} \sum_{i=1}^{N L}\left[\left\langle\gamma_{Y}^{M}(i \Delta r)\right\rangle-\left\langle\gamma_{Y}^{M-1}(i \Delta r)\right\rangle\right]^{2}\right\}^{1 / 2} \leq \varepsilon
$$

where $\Delta r$ is the discretization used for the two points lag, $N L=L / \Delta x$ is the number of points in which the maximum lag $L$ has been divided, $\left\langle\gamma_{Y}^{M}(i \Delta r)\right\rangle$ is the expected value of the semivariogram computed with reference to $M$ Monte Carlo 

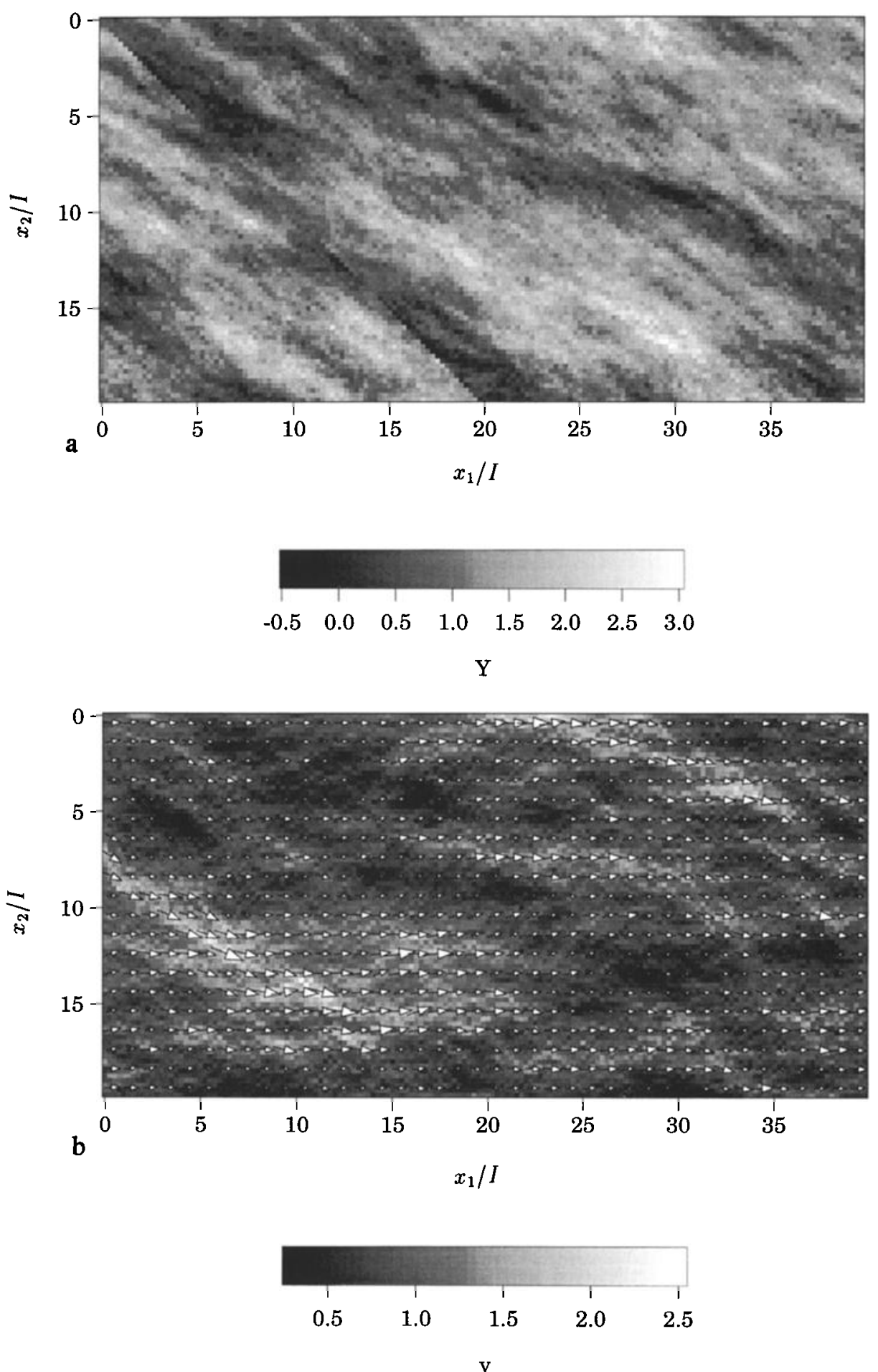

Figure 1. (a) An example of $\log$ transmissivity field for $a=0.1, \beta=0.75$ and (b) the resulting velocity field.

realizations, and $\varepsilon$ is the average residual error. Assuming $\varepsilon=$ $10^{-6}$, convergence has always been obtained with fewer than about 1500 realizations, which are required for the convergence of the second-order moments. The log conductivity variance depends on $\beta$ through (7). Hence, to assess the impact of $\beta$ on transport, independently from the value of $\sigma_{Y}^{2}$, we use $a=0.0025$ for the case $\beta=1.75$, which, for the domain dimensions used in this paper, shows values of $\sigma_{Y}^{2}$ of the same order of magnitude as that observed for the case $\beta=0.75$ and $a=0.1$. The matching between the expected value of the a posteriori computed semivariogram and (1) is good for both cases $\beta=0.75$ and $\beta=1.75$. The interval of confidence increases as $\beta$ increases as a consequence of the lack of ergodicity; see Figure $2 b$. More refined testing of the simple scaling assumption postulated by (1) has been addressed by studying the scaling properties of the generalized semivariogram $\gamma_{q}(r)$ $=\left\langle|Y(\mathbf{x}+\mathbf{r})-Y(\mathbf{x})|^{q}\right\rangle_{r=|\mathbf{r}|}$, which should scale as $C_{q}^{\gamma}(r) \propto$ $r^{q \beta / 2}$ [Lavallèe et al., 1993]. Random fields numerically gener- 

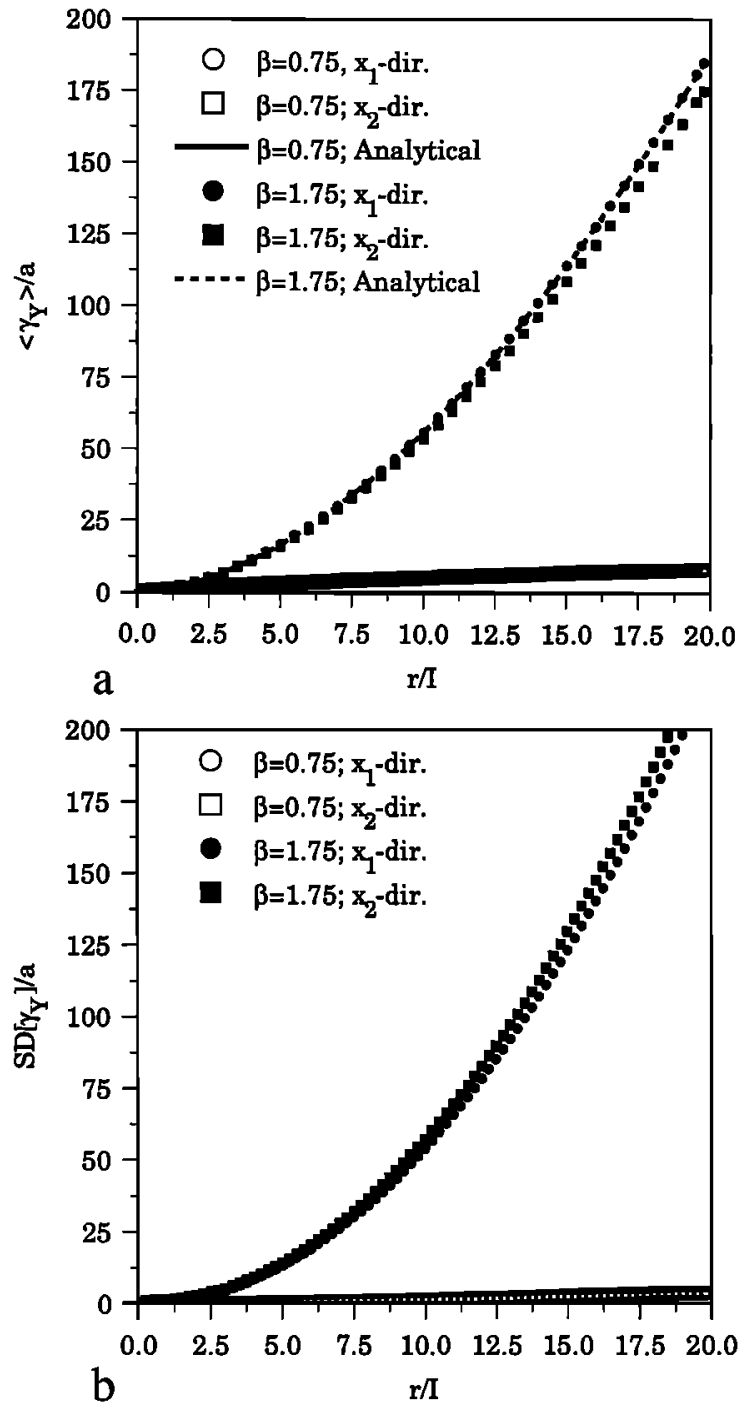

Figure 2. Log transmissivity semivariogram; $\beta=0.75, a=$ 0.1 and $\beta=1.75, a=0.0025$ : (a) expected value $\left\langle\gamma_{Y}\right\rangle$; (b) standard deviation $\operatorname{SD}\left[\gamma_{Y}\right]$.

ated by the method of Bellin and Rubin [1996] yield acceptable scaling properties for our purposes.

The impact of boundary conditions on transport should be carefully evaluated prior to comparing numerical and analytical solutions, the latter being obtained in infinite domains. A comprehensive theoretical analysis of the influence of Cauchy and Neumann boundary conditions on velocity statistics in a semi-infinite domain for a stationary single-scale log conductivity field characterized by an exponential covariance function has been carried out by Rubin and Dagan [1988, 1989]. The conclusion of the above study is that the influence of the boundary conditions becomes insignificant at a distance larger than three integral scales from the boundary. In a numerical work Bellin et al. [1992] suggested that for large field dimensions and $L_{2} / L_{1}=2$, the distance from the boundaries beyond which the flow field is not affected by the boundary conditions increases with the log conductivity variance. However, the limit of about three log conductivity integral scales is acceptable in the explored range of the log conductivity variance, that is, $\sigma_{Y}^{2}<1.6$. Recently, Osnes [1995] suggested that in the transverse direction (i.e., the direction normal to the impervious boundaries and the mean flow), the head variance $\sigma_{H}^{2}$ is constant with good approximation for $L_{2} / L_{1}<2 / 3$. For $L_{2} / L_{1}>2 / 3$ the variance $\sigma_{H}^{2}$ increases from the center of the domain to the impervious boundaries. For a fixed value of the ratio $L_{2} / L_{1}$ the finite domain solution approaches the solution obtained for an unbounded domain as $L_{1}$ increases. Furthermore, as an effect of the smoothing introduced by the Poisson operator, $\sigma_{H}^{2}$ decreases as $L_{2} / L_{1}$ increases.

For log conductivity fields characterized by model (1) the integral scale increases linearly with the domain dimension (see (8)), and then boundary conditions are expected to influence the velocity field irrespective of domain dimensions.

Figures $3 \mathrm{a}$ and $3 \mathrm{~b}$ show the longitudinal velocity variance along the sections $x_{2}=L_{2} / 2$, in longitudinal direction, and $x_{1}=L_{1} / 2$, in transverse direction, for $\beta=0.75$ and $\beta=1.75$, respectively. Here, $\sigma_{u}^{2}$ is divided by $L_{2}^{\beta}$, which is proportional to the $\log$ conductivity field variance. Furthermore, distances are dimensionless with respect to $L_{1}$ and $L_{2}$ for the longitudinal and transverse directions, respectively. While in the longitudinal direction $\sigma_{u}^{2} / a U^{2} L_{2}^{\beta}$ is nearly constant, in the transverse direction it assumes a parabolic shape slightly dependent
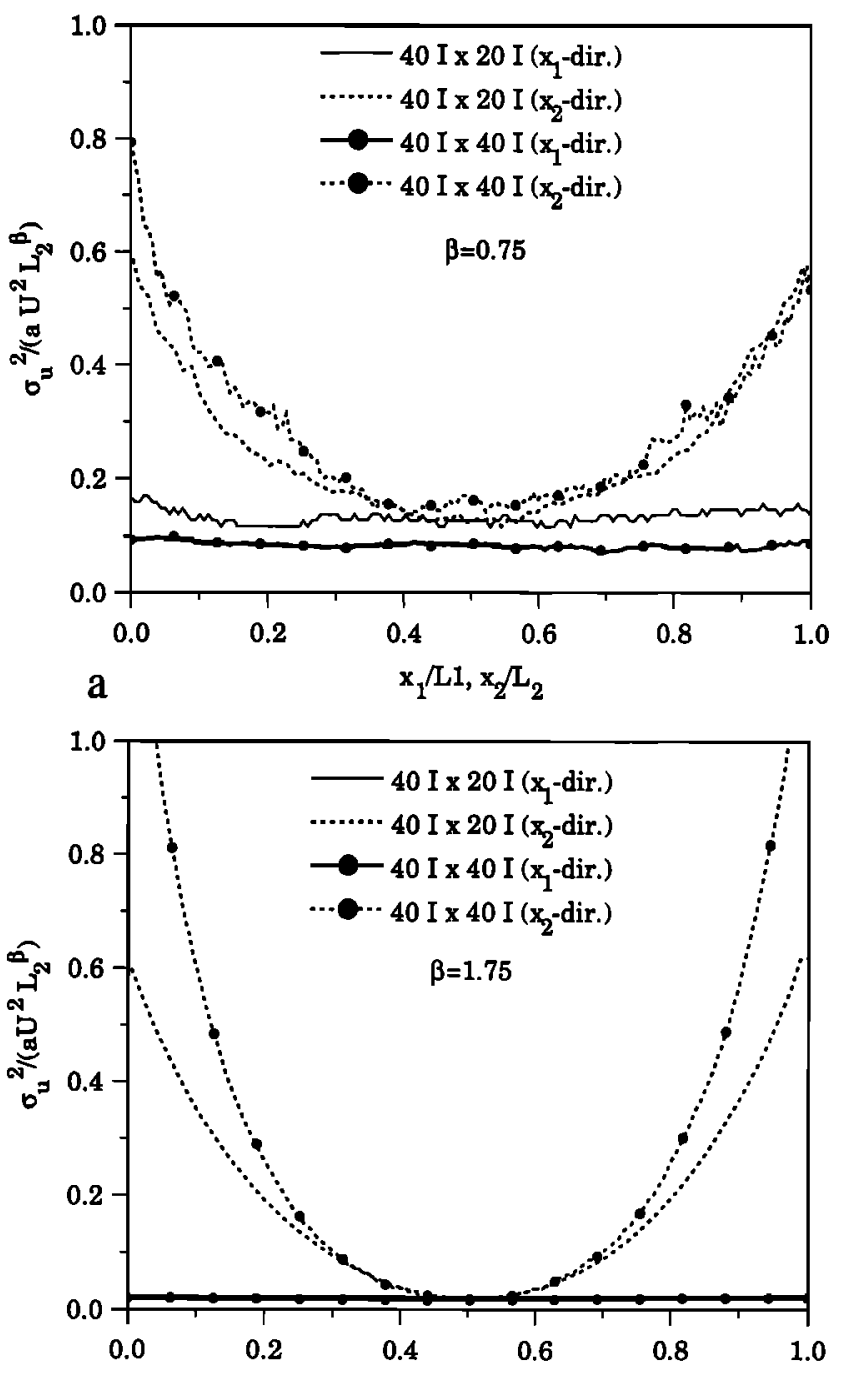

b

$\mathrm{x}_{1} / \mathrm{L}_{1} ; \mathrm{x}_{2} / \mathrm{L}_{2}$

Figure 3. Longitudinal velocity variance along the sections $x_{2}=L_{2} / 2$ and $x_{1}=L_{1} / 2$ for (a) $\beta=0.75, a=0.1$ and (b) $\beta=1.75, a=0.0025$. 
on the field dimension. The longitudinal velocity variance is maximum at the impervious boundaries and decreases in transverse direction with the distance from the boundaries reaching the minimum value at the domain center. This is in agreement with the findings of Osnes [1995] obtained for single-scale heterogeneous media and characteristic domain dimensions of few integral scales. Since the integral scale increases linearly with the domain dimension (see (8)), the velocity field depends on the boundary conditions irrespective of domain dimensions. Hence an inner core region in which the velocity field is independent on the boundary conditions cannot be strictly defined and the solutions are expected to depend on the cutoff. Figures $4 \mathrm{a}$ and $4 \mathrm{~b}$ show $\gamma_{u}(\mathbf{r})$ for $\beta=0.75$ and $\beta=1.75$. They depend on the velocity variance from the "infrared" cutoff imposed by the domain size. However, as also shown in Figures $4 a$ and $4 b$, the domain dimension has a relatively small influence on the difference $\gamma_{u_{1}}(r, 0)-\gamma_{u_{1}}(0, r)$ such that according to (A1),
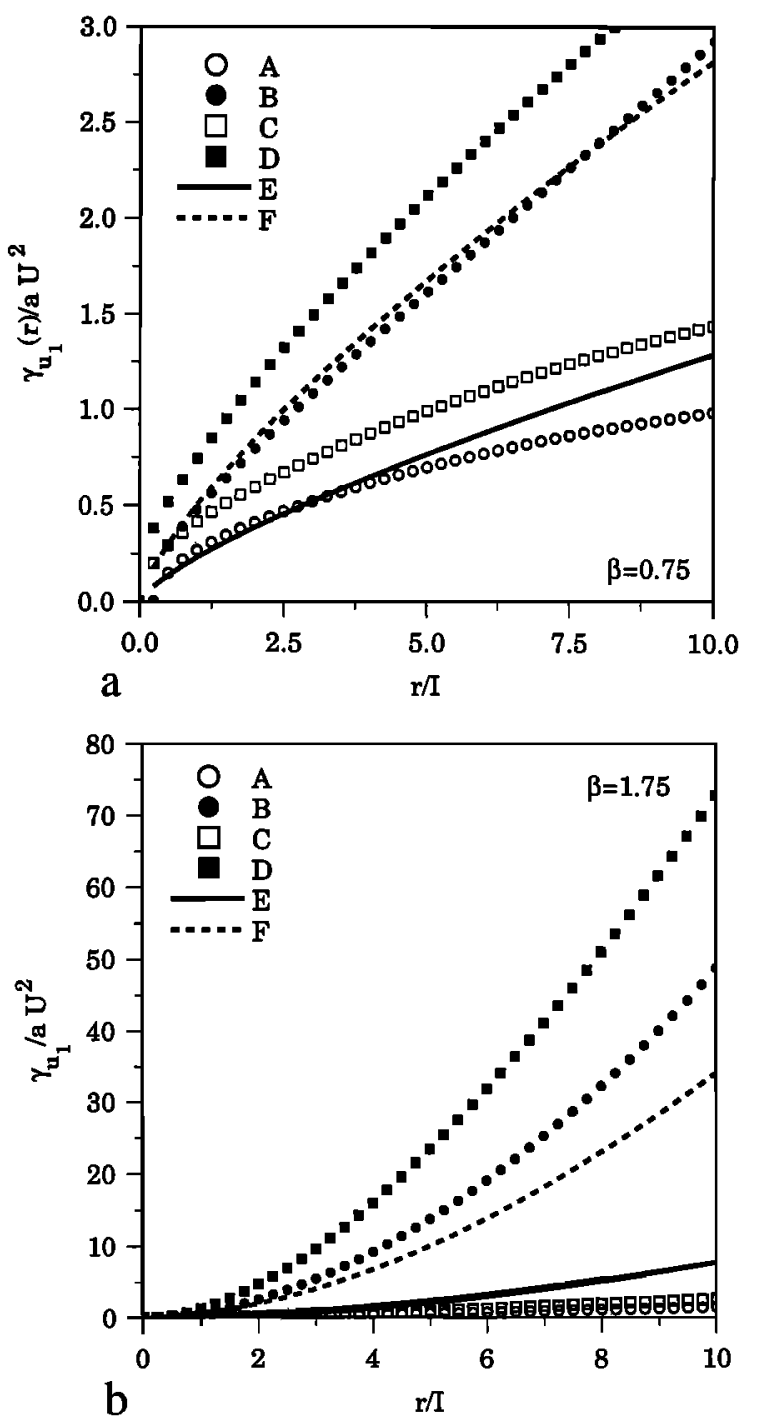

Figure 4. Longitudinal velocity semivariogram for (a) $\beta=$ $0.75, a=0.1$ and (b) $\beta=1.75, a=0.0025$. In all cases: $\mathrm{A}, \gamma_{u}(r, 0)$ and domain dimensions $40 I \times 20 I ; \mathrm{B}, \gamma_{u}(0, r)$ and domain dimensions $40 I \times 20 I ; \mathrm{C}, \gamma_{u}(r, 0)$ and domain dimensions $40 I \times 40 I ; \mathrm{D}, \gamma_{u}(0, r)$ and domain dimensions $40 I \times 40 I ; \mathrm{E}, \gamma_{u}(r, 0)$ given by equation (A2); $\mathrm{F}, \gamma_{u}(0, r)$ given by equation (A2). the effective dispersion coefficient is expected to depend weakly on the actual cutoff.

\section{Discussion}

As is customary in stochastic approaches [e.g., Dagan, 1989], we use the robust description yielded in terms of plume moments which are computed as follows: (1) the trajectory of the plume centroid:

$$
R_{t}^{v}(t)=\frac{1}{N} \sum_{k=1}^{N} X_{t}^{k, v}(t) \quad i=1,2
$$

where $X_{i}^{k, v}$ is the $i$ th component of the trajectory of the $k$ th particle for the realization $v$ and $N$ is the number of particles; (2) the second-order central plume moment:

$$
S_{l y}^{v}(t)=\frac{1}{N-1} \sum_{k=1}^{N}\left(\left[X_{l}^{k, v}(t)-R_{l}^{v}(t)\right]\left[X_{J}^{k, v}(t)-R_{J}^{v}(t)\right]\right)
$$

The moments (10) and (11) are computed in each realization $v$. They represent the overall plume behavior in one of the (equally probable) $\log$ transmissivity fields. Expected values and variances of $R$, and $S_{\imath \jmath}$ are obtained taking expectation over $M$ independent Monte Carlo realizations:

$$
\begin{gathered}
\left\langle R_{l}(t)\right\rangle=\frac{1}{M} \sum_{v=1}^{M} R_{l}^{v}(t) \\
R_{t i}(t)=\operatorname{Var}\left(R_{\imath}(t)\right)=\frac{1}{M-1} \sum_{v=1}^{M}\left[R_{\imath}^{v}(t)-\left\langle R_{l}(t)\right\rangle\right]^{2} \\
\left\langle S_{v j}(t)\right\rangle=\frac{1}{M} \sum_{v=1}^{M} S_{\imath \jmath}^{v}(t) \\
\operatorname{Var}\left(S_{t j}(t)\right)=\frac{1}{M-1} \sum_{v=1}^{M}\left[S_{v j}^{v}(t)-\left\langle S_{l j}(t)\right\rangle\right]^{2}
\end{gathered}
$$

The expected values (12) and (14) represent the most probable estimate of the actual plume behavior. The variances (13) and (15) can be used in order to identify the range of variability of the actual plume statistics around the expected value. A null variance implies that the plume is in ergodic conditions and in each realization the spatial moments (10) and (11) resemble the expected value, irrespective of the actual distribution of hydraulic transmissivity. In formations such as those represented by (1) the ergodic condition cannot be reached and the practical consequence is that the uncertainties in estimating the actual plume moments increase with the transverse plume dimension. Hence $\left\langle R_{z}(t)\right\rangle$ and $\left\langle S_{i j}(t)\right\rangle$ become progressively less reliable in estimating the actual plume behavior as time (since plume injection) increases. The computation of the interval of confidence for the estimations provided by the expected values of the plume moments is then of paramount importance. Expected values and variances are computed with reference to $M=1500$ Monte Carlo realizations, which has been suggested to suffice in ensuring convergence [Bellin et al., 1992].

The numerical expected value of the trajectory of the plume 

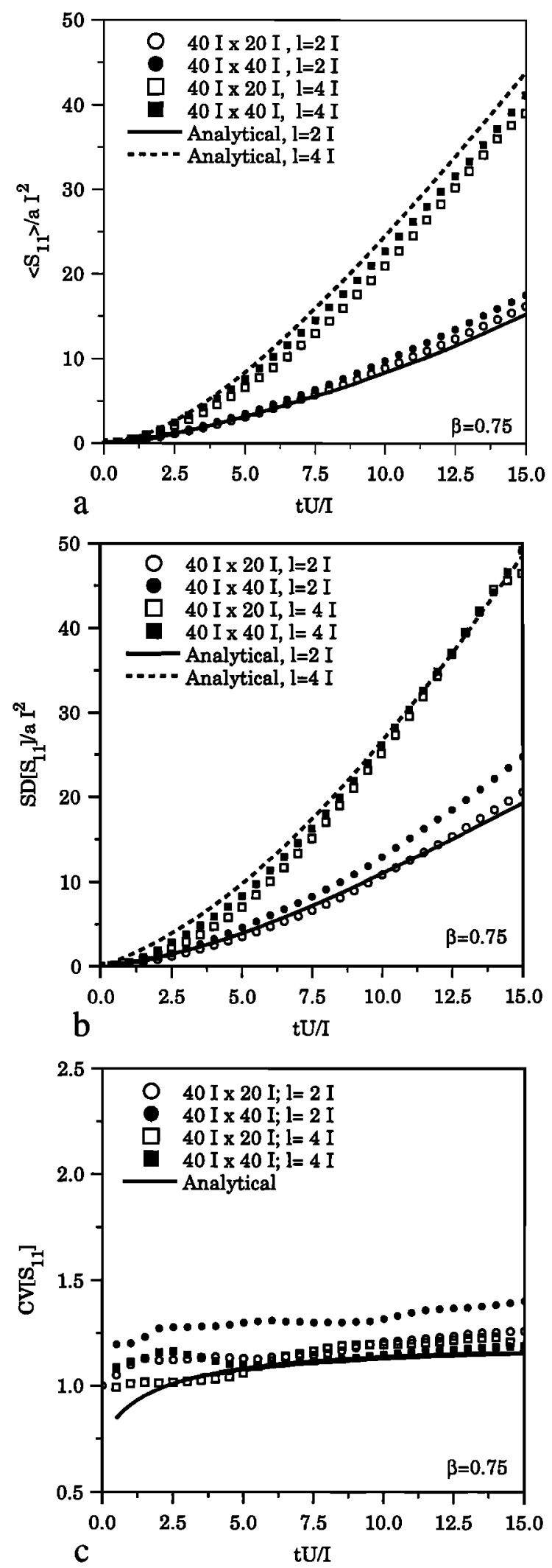

Figure 5. (a) Expected value of the longitudinal second order moment $S_{11}$. (b) The standard deviation of $S_{11}$. (c) The coefficient of variation of $S_{11}$ for $l=2 I$ and $4 I$ and field dimensions $40 I \times 20 I$ and $40 I \times 40 I$. In all cases $\beta=0.75$, $a=0.1$. centroid is in good agreement with the analytical solution: $\langle\mathbf{R}(t)\rangle=\mathbf{U} t$.

Figures 5a and 5b show the expected value of the secondorder longitudinal spatial plume moment $\left\langle S_{11}\right\rangle$ and its standard deviation $\operatorname{SD}\left[S_{11}\right]$, respectively. Simulations are performed with $l=2 I$ and $4 I$ and $\beta=0.75$ employing a maximum dimensionless simulation time of 15 .

Numerical and analytical solutions of $\left\langle S_{11}\right\rangle$ and $\operatorname{SD}\left[S_{11}\right]$ are in good agreement both for $l=2 I$ and $l=4 I$. Figure $5 \mathrm{c}$ shows numerical and analytical solutions for $\operatorname{CV}\left[S_{11}\right]=\operatorname{SD}\left[S_{11}\right] /$ $\left\langle S_{11}\right\rangle$. The matching between analytical and numerical solutions is acceptable, and $\mathrm{CV}\left[S_{11}\right]$ is slowing varying in time, assuming values inside the range $0.9 \div 1.3$ over the explored range of time. The practical result is that in actual plumes the longitudinal spreading may differ more than $100 \%$ from values given by (A4). The actual $S_{11}$ fluctuates in time around $\left\langle S_{11}\right\rangle$ leading to the possibility to infer $\left\langle S_{11}\right\rangle$ from measurements of $S_{11}$ at several time steps [Dagan, 1991]. The moving average of the actual second-order longitudinal plume moment is in fact characterized by a smaller coefficient of variations (G. Dagan, personal communication, 1996). It still holds, however, that (A4) provides the best estimate of the actual spreading. In agreement with the first-order analytical solution, $\left\langle S_{11}\right\rangle$ increases as a power of the transverse initial plume dimension $l$. Such behavior stems from the increased chances for plumes with larger initial dimensions to sample zones with higher contrast in hydraulic transmissivity. Here, $\left\langle S_{11}\right\rangle$ scales as $\left\langle S_{11}\right\rangle \propto(U t / I)^{\gamma}$ with $\gamma<1$, and then the occurrence of situations of anomalous dispersion seems improbable. In such media the plume is not ergodic irrespective of the initial transverse plume dimension which, on the other hand, strongly influences the second-order plume moment.

In order to test the case in which anomalous dispersion is expected to develop, we repeated the numerical simulations using the semivariogram model (1) with $\beta=1.75$ and $a=$ 0.0025 . As in the case $\beta=0.75$, the simulations were repeated for two different domains with the same longitudinal dimension $\left(L_{1}=40 I\right)$ and transverse dimensions equal to $L_{2}=20 I$ and $40 I$, respectively.

The values of $\left\langle S_{11}\right\rangle, \operatorname{SD}\left[S_{11}\right]$, and $\operatorname{CV}\left[S_{11}\right]$ are shown in Figures $6 \mathrm{a}, 6 \mathrm{~b}$, and $6 \mathrm{c}$, respectively. $\left\langle S_{11}\right\rangle$ and $\mathrm{SD}\left[S_{11}\right]$ obtained by the numerical analysis slightly overestimate the analytical solutions provided by (A4) and (A13). Besides the small differences in the analytical and numerical results for $\left\langle S_{11}\right\rangle$, the slopes of the two curves are in a good agreement. Therefore we conclude that the asymptotic values of analytical and numerical longitudinal dispersion coefficients agree well. Furthermore $\left\langle S_{11}\right\rangle$ scales as $\left\langle S_{11}\right\rangle \propto(U t / I)^{\gamma}$, where $\gamma$ is a scaling exponent larger than 1 . In the analyzed range of variability of the log conductivity field (i.e., field variances limited to about 2), we concur with the theoretical results [Dagan, 1994] that the dispersion is anomalous for $\beta>1$ [Dagan, 1994] and Fickian for $\beta \leq 1$.

Figures $7 \mathrm{a}$ and $7 \mathrm{~b}$ show $X_{11} / L_{2}^{\beta}$ as function of the dimensionless plume travel distance for $\beta=0.75$ and 1.75 , respectively. $X_{11}$ is about 1 order of magnitude larger than $\left\langle S_{11}\right\rangle$, and in all cases the expression (4) holds. Since $X_{11}$ scales as $L_{2}^{\gamma}$, with $\gamma>1$, we conclude in agreement with Glimm et al. [1993] that the ergodic second-order plume moments show a strong dependence on the cutoff. This is not the case for the nonergodic second-order moment $\left\langle S_{11}\right\rangle$ which is weakly affected by the imposed "infrared" cutoff. 

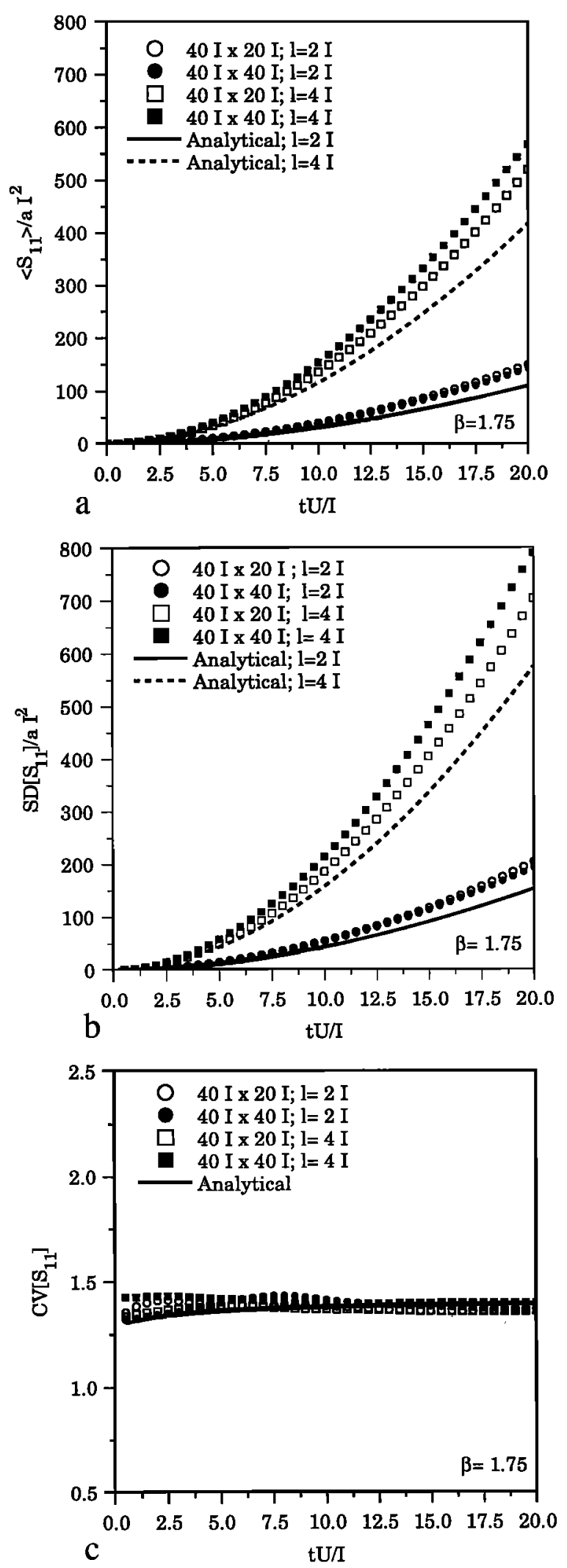

Figure 6. (a) Expected value of the longitudinal second order moment $S_{11}$. (b) The standard deviation of $S_{11}$. (c) The coefficient of variation of $S_{11}$ for $l=2 I$ and $4 I$ and field dimensions $40 I \times 20 I$ and $40 I \times 40 I$. In all cases $\beta=1.75$, $a=0.0025$.
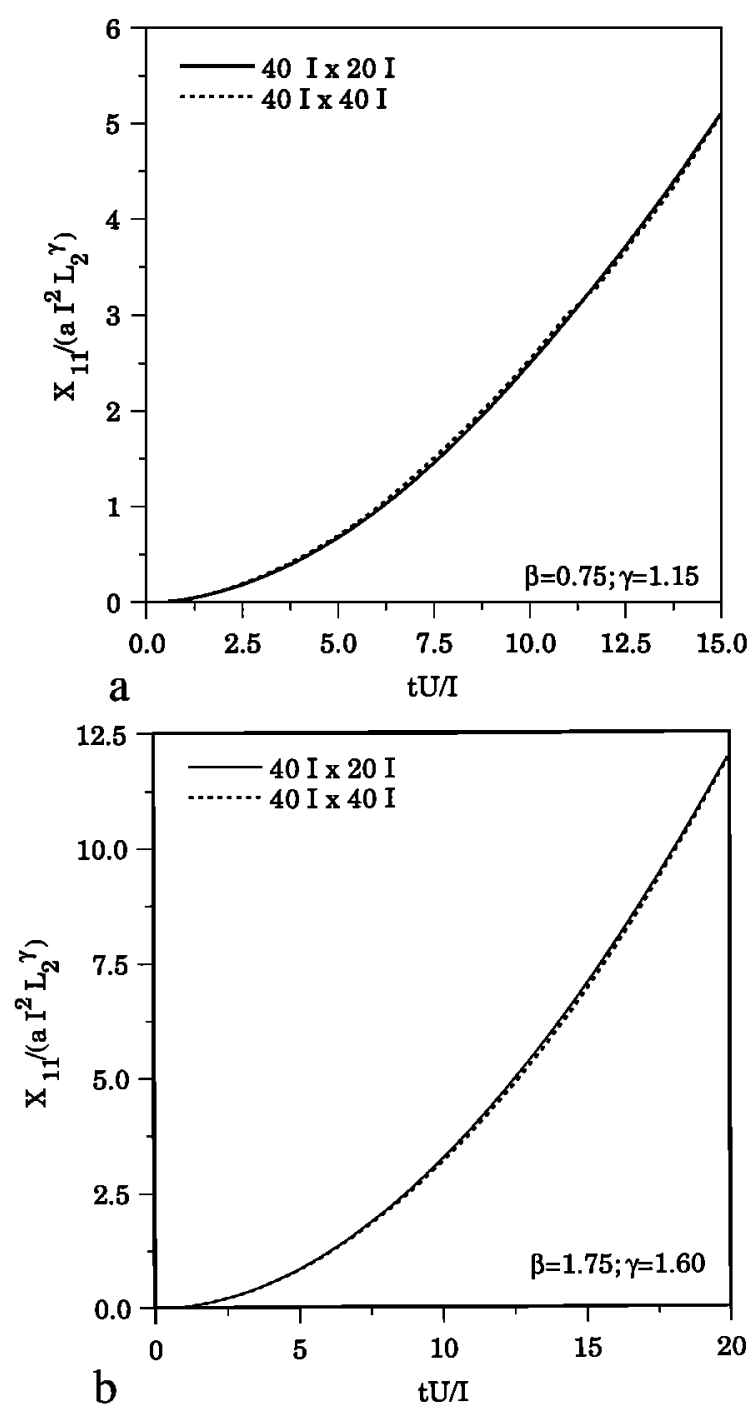

Figure 7. Ergodic second-order plume moment $X_{11}$ for different field dimensions; (a) $\beta=0.75, a=0.1$ and (b) $\beta=$ $1.75, a=0.0025$.

\section{Conclusions}

The following main conclusions are worth mentioning: First, novel analytical solutions for the effective longitudinal coefficient of macrodispersion $D_{L}$ and the expected value of the second-order longitudinal plume moment $\left\langle S_{11}\right\rangle$ are proposed. These are derived in a stationary increment $\log$ transmissivity field characterized by a power law semivariogram: $\left(\gamma_{Y}(r)=a\right.$ $\left.r^{\beta}\right)$ and support, and slightly extend, the solutions proposed by Dagan [1994]. Our analytical solutions confirm Dagan's conclusions concerning the occurrence of anomalous dispersion which develops for $\beta \geq 1$ while for $\beta<1$ the transport proves Fickian. We have also proposed analytical solutions for $\operatorname{SD}\left[S_{11}\right]$, which is a measure of the interval of confidence of the estimate of the actual spreading provided by $\left\langle S_{11}\right\rangle$.

Second, we have also relaxed the linearity assumptions and solved numerically the fully nonlinear flow and transport problem. For $\beta<1$ we found a good agreement between analytical and numerical solutions. For large $\beta$ (i.e., $\beta=1.75$ ) the numerical solutions slightly overestimate the analytical values. The anomalous dispersion behavior predicted by the analytical 
solution is, however, confirmed by our numerical results at least in the explored range of variability of the log conductivity field variance $\left(\sigma_{Y}^{2}<2\right)$. Furthermore, the effective dispersion coefficient is not affected by the distance cutoff which results from the use of a finite field dimension in the numerical analysis.

As a results of the good match for both $\left\langle S_{11}\right\rangle$ and $\operatorname{SD}\left[S_{11}\right]$, a good agreement is observed between analytical and numerical solutions for $\mathrm{CV}\left[S_{11}\right]$. In the explored range of travel times the values of $\mathrm{CV}\left[S_{11}\right]$ remain close to 1 . This finally suggests that in actual plumes the effective dispersion could be overestimated (or underestimated) by an amount close to the expected value. We conclude that because of the large value of $\mathrm{CV}\left[S_{11}\right]$ and in absence of conditioning with log conductivity measurements, caution needs to be posed in the interpretation of large-scale tracer tests in evolving scale formations.

\section{Appendix: Analytical Solutions}

In fields characterized by a semivariogram given by (1), the $\log$ conductivity variance $\sigma_{Y}^{2}$ and then also the mean velocity, $\mathrm{U}$; the velocity covariance function, $C_{u}$; and $d_{L}$ are not defined. According to the approach employed by Dagan [1994] the definition of an "infrared cutoff," that is, the filtering out of wavelengths larger than the characteristic field dimension, is a sufficient condition to render $\sigma_{Y}^{2}, \mathrm{U}$, and $C_{u}$ finite. Under the above hypothesis, and for solute injection along a strip of length $l$ normal to the mean flow direction, the effective dispersion coefficient assumes the following expression [Dagan, 1994]:

$$
D_{L}(t)=\frac{2}{U l^{2}} \int_{0}^{l} \int_{0}^{t U}\left(l-r_{2}\right)\left[\gamma_{u}\left(r_{1}, r_{2}\right)-\gamma_{u}\left(r_{1}, 0\right)\right] d r_{1} d r_{2}
$$

where $\gamma_{u}$ is the velocity semivariogram. Interestingly, (A1) does not depend on $\mathbf{U}$, the assumption being that $\mathbf{U}$ is finite and constant. Changes of $\mathbf{U}$ result in different paths for the plume center of mass with the actual spreading that remains unchanged. The values of $\gamma_{u}$ at first order are given by [Dagan, 1994]:

$$
\begin{aligned}
\gamma_{u}\left(r_{1}, r_{2}\right)= & \frac{U^{2} a}{(2+\beta)(4+\beta)}\left[(1+\beta)(3+\beta) r^{\beta}\right. \\
& \left.-2 \beta(1+\beta) r_{1}^{2} r^{\beta-2}+\beta(\beta-2) r_{1}^{4} r^{\beta-4}\right]
\end{aligned}
$$

where $r=\left(r_{1}^{2}+r_{2}^{2}\right)^{1 / 2}$. The solution of (A1) has been obtained by Dagan [1994] with $\gamma_{u}$ given by (A2) and employing numerical quadrature.

Replacing $\gamma_{u}$ in (A1) with (A2) and performing an analytical quadrature, we obtain the following expression:

$$
\begin{aligned}
& \frac{D_{L}(t)}{U a l^{1+\beta}}=-\frac{3}{(1+\beta)(2+\beta)(4+\beta)} x^{1+\beta} \\
& -\frac{2}{(2+\beta)^{2}(3+\beta)(4+\beta)} x^{3+\beta} \\
& +\left\{\left[6(3+\beta) x_{2} F_{1}\left(1 / 2,-\beta / 2,3 / 2,-x^{2}\right)\right\}\right. \\
& \left.\left.+2 x_{2}^{3} F_{1}\left(3 / 2,-\beta / 2,5 / 2,-x^{2}\right)\right] /\left[3(2+\beta)^{2}(4+\beta)\right]\right\}
\end{aligned}
$$

where $x=t U / l=L / l$ is the dimensionless mean travel distance and ${ }_{2} F_{1}(a, b ; c, z)$ is the hypergeometric function [e.g., Gradshteyn and Ryzhik, 1980].
The integration in time yields the following expression for the effective longitudinal second-order centered plume moment:

$$
\begin{aligned}
\frac{\left\langle S_{11}(t)\right\rangle}{a l^{2+\beta}=} & \frac{\left(-2 x^{2+\beta}\left(36+21 \beta+3 \beta^{2}+2 x^{2}+2 \beta x^{2}\right)\right.}{(1+\beta)(2+\beta)^{2}(3+\beta)(4+\beta)^{2}} \\
& -\frac{1}{3(2+\beta)^{2}(4+\beta)^{2}}[-60-12 \beta \\
& +4\left(1+x^{2}\right)^{\beta / 2}\left(15+3 \beta+18 x^{2}+3 \beta x^{2}+3 x^{4}\right) \\
& -4\left(36+21 \beta+3 \beta^{2}\right) x_{2}{ }_{2} F_{1}\left(1 / 2,-\beta / 2,3 / 2,-x^{2}\right) \\
& \left.-4(4+\beta) x_{2}^{4} F_{1}\left(3 / 2,-\beta / 2,5 / 2,-x^{2}\right)\right]
\end{aligned}
$$

According to (A3), the effective dispersion grows with a power law which depends on $\beta$.

For large $x$ the effective dispersion coefficient grows as

$$
\begin{aligned}
D_{L}(t) \simeq & U a l^{2} \frac{15 \beta}{12(2+\beta)(4+\beta)(\beta-1)} x^{\beta-1} \\
& +O\left(U a l^{1+\beta}\right)
\end{aligned}
$$

Equation (A5) coincides with the limit for large travel time obtained by Dagan [1994] using a different approach.

As suggested by Dagan [1994], (A5) implies that the behavior is Fickian for $0<\beta<1$ and anomalous for $1 \leq \beta \leq 2$. For the limit case $\beta=1$, (A3) becomes

$$
\begin{aligned}
\frac{D_{L}(t)}{U a l^{2}}= & -\frac{x^{2}}{10}-\frac{x^{4}}{90}+\left(1+x^{2}\right)^{1 / 2}\left[\frac{17}{180} x+\frac{x^{3}}{90}\right] \\
& +\frac{1}{12} \operatorname{arcsinh}(x)
\end{aligned}
$$

For large $x,(\mathrm{~A} 6)$ behaves as $D_{L} \simeq O\left[U a l^{2} \operatorname{arcsinh}(x) / 12\right]$. The dispersion is thus anomalous but with a logarithmic growing rate. Correspondingly, $\left\langle S_{11}\right\rangle$ assumes the following expression:

$$
\begin{aligned}
\frac{\left\langle S_{11}(t)\right\rangle}{a l^{3}} & =-\frac{x^{3}}{15}-\frac{x^{5}}{225}+\left(1+x^{2}\right)^{1 / 2}\left[-\frac{8}{75}+\frac{29}{450} x^{2}+\frac{x^{4}}{225}\right] \\
& +\frac{x}{6} \operatorname{arcsinh}(x)+\frac{8}{75}
\end{aligned}
$$

For large mean travel distances, $\left\langle S_{11}\right\rangle$ behaves like $\left\langle S_{11}\right\rangle=$ $O\left[a l^{3} x \operatorname{arcsinh}(x) / 6\right]$.

For $\beta=2 D_{L}$ and $\left\langle S_{11}\right\rangle$ are given by

$$
\frac{D_{L}(t)}{a l^{3}}=\frac{5}{48} x \quad \frac{\left\langle S_{11}(t)\right\rangle}{a l^{4}}=\frac{5}{48} x^{2}
$$

Under the aforementioned linearity hypotheses the variance of the longitudinal plume moment (for solute injection along a strip of length $l$ normal to the mean flow direction) assumes the following expression [Dagan, 1990]:

$$
\begin{aligned}
\operatorname{Var}\left[S_{11}(t)\right]= & 2 R_{11}^{2}(x)+2 \int_{0}^{1} \int_{0}^{1} X_{11}^{2}\left(x, a^{\prime}-a^{\prime \prime}\right) d a^{\prime} d a^{\prime \prime} \\
& -4 \int_{0}^{1} \int_{0}^{1} \int_{0}^{1} X_{11}\left(x, a^{\prime}-a^{\prime \prime}\right) \\
& \cdot X_{11}\left(x, a^{\prime \prime}-a^{\prime \prime \prime}\right) d a^{\prime} d a^{\prime \prime} d a^{\prime \prime \prime}
\end{aligned}
$$


where $X_{11}\left(x, a^{\prime}\right)$ is the particle trajectory variance for the particle injected at time $t=0$ at position $\left(0, a^{\prime}\right)$. Here space coordinates are made dimensionless with $l$. It is possible to show that $\operatorname{Var}\left[S_{11}\right]$ does not depend on $\sigma_{u}^{2}$; that is, the cutoff imposed at the log conductivity field does not modify the plume moments which are then influenced solely by the scales sampled by the plume. Therefore $\operatorname{Var}\left[S_{11}(x)\right]$ assumes the following expression:

$$
\begin{aligned}
& \operatorname{Var}\left[S_{11}(t)\right]=2 R_{\gamma, 11}^{2}(x) \\
& +2 \int_{0}^{1} \int_{0}^{1} X_{\gamma, 11}^{2}\left(x, a^{\prime}-a^{\prime \prime}\right) \cdot d a^{\prime} d a^{\prime \prime} \\
& \quad-4 \int_{0}^{1} \int_{0}^{1} \int_{0}^{1} X_{\gamma, 11}\left(x, a^{\prime}-a^{\prime \prime}\right) \\
& \cdot X_{\gamma, 11}\left(x, a^{\prime \prime}-a^{\prime \prime \prime}\right) d a^{\prime} d a^{\prime \prime} d a^{\prime \prime \prime}
\end{aligned}
$$

where the subscript $\gamma$ means that the referenced quantity is computed replacing the velocity correlation function with its semivariogram into the corresponding equations.

The covariances $X_{\gamma, 11}(x, b)$ and $R_{\gamma, 11}(x)$ are given by

$$
\begin{aligned}
\frac{X_{\gamma, 11}(x, b)}{a l^{2+\beta}}= & \frac{2 \int_{0}^{t}(t-\tau) \gamma_{u}(U \tau, b) d \tau}{a l^{2+\beta}} \\
= & \frac{2}{15(2+\beta)^{2}(4+\beta)\left(x^{2}+b^{2}\right)} \\
& \cdot\left\{-15\left(x^{2}+b^{2}\right)^{\beta / 2}\left(3 x^{4}+18 x^{2} b^{2}+6 \beta x^{2} b^{2}\right.\right. \\
& \left.+15 b^{4}+8 \beta b^{4}+\beta^{2} b^{4}\right)+|b|^{\beta}\left[225 x^{2} b^{2}\right. \\
& +120 \beta x^{2} b^{2}+15 \beta^{2} x^{2} b^{2}+225 b^{4}+120 \beta b^{4} \\
& +15 \beta^{2} b^{4}+15\left(6+11 \beta+6 \beta^{2}+\beta^{3}\right) x^{2} \\
& \cdot\left(x^{2}+b^{2}\right)_{2} F_{1}\left(1 / 2,-\beta / 2,3 / 2,-(x / b)^{2}\right) \\
& -10 \beta\left(2+3 \beta+\beta^{2}\right) x^{4}\left(x^{2}+b^{2}\right) \frac{1}{b^{2}}{ }_{2} F_{1}(3 / 2,1 \\
& \left.-\beta / 2,5 / 2,-(x / b)^{2}\right)+3 \beta\left(\beta^{2}-4\right) x^{6}\left(x^{2}\right. \\
& \left.\left.\left.+b^{2}\right) \frac{1}{b^{4}}{ }_{2} F_{1}\left(5 / 2,2-\beta / 2,7 / 2,-(x / b)^{2}\right)\right]\right\}
\end{aligned}
$$

$$
\begin{aligned}
& \frac{R_{\gamma, 11}(x)}{a l^{2+\beta}}=\frac{1}{l^{4+\beta}} \int_{V_{0}} X_{11}\left(x, a^{\prime}-a^{\prime \prime}\right) d a^{\prime} d a^{\prime \prime}=4\left\{-\left[\left(x^{4+\beta}\right)\right.\right. \\
& \left./\left[(2+\beta)^{2}(3+\beta)(4+\beta)^{2}\right]\right]+[[15+3 \beta \\
& \left.\quad-\left(1+x^{2}\right)^{\beta / 2}\left(15+3 \beta+18 x^{2}+3 \beta x^{2}+3 x^{4}\right)\right] \\
& \left./\left[3(2+\beta)^{2}(4+\beta)^{2}\right]\right]+\left[\left[x^{2}\left(36+21 \beta+3 \beta^{2}\right)_{2} F_{1}(1 / 2,\right.\right. \\
& \left.-\beta / 2,3 / 2,-x^{2}\right)+x^{4}(4+\beta)_{2} F_{1}(3 / 2,-\beta / 2,5 / 2, \\
& \left.\left.\left.\left.-x^{2}\right)\right] /\left[3(2+\beta)^{2}(4+\beta)^{2}\right]\right]\right\}
\end{aligned}
$$

in which $b$ is the dimensionless separation distance, perpendicular to the mean flow, of two points of the initial plume.

With reference to (A10), and considering the symmetry of $X_{11}$ with respect to the separation distance, the standard deviation $\mathrm{SD}\left[S_{11}\right]$ assumes the following expression:

$$
\mathrm{SD}\left[S_{11}(t)\right]=\left[2 R_{\gamma, 11}^{2}(x)+2 I_{1}-4 I_{2}\right]^{1 / 2}
$$

Correspondingly, the coefficient of variation is given by

$$
\operatorname{CV}\left[S_{11}\right]=\frac{\operatorname{SD}\left[S_{11}\right]}{\left\langle S_{11}\right\rangle}
$$

where $I_{1}$ and $I_{2}$ are given by

$$
\begin{gathered}
I_{1}=\int_{0}^{1} \int_{0}^{1} X_{\gamma, 11}^{2}\left(x, a^{\prime}-a^{\prime \prime}\right) d a^{\prime} d a^{\prime \prime} \\
=2 \int_{0}^{1}(1-b) X_{\gamma_{1} 11}^{2}(x, b) d b \\
I_{2}=\int_{0}^{1} \int_{0}^{1} \int_{0}^{1} X_{\gamma_{0} 11}\left(x, a^{\prime}-a^{\prime \prime}\right) X_{\gamma, 11}\left(x, a^{\prime \prime}-a^{\prime \prime \prime}\right) d a^{\prime} d a^{\prime \prime} d a^{\prime \prime \prime} \\
=2 \int_{0}^{1} \int_{0}^{1-b^{\prime}}\left(1-b^{\prime}-b^{\prime \prime}\right) X_{\gamma, 11}\left(x, b^{\prime}\right) X_{\gamma, 11}\left(x, b^{\prime \prime}\right) d b^{\prime \prime} d b^{\prime} \\
+4 \int_{0}^{1} \int_{0}^{b^{\prime}}\left(1-b^{\prime}\right) X_{\gamma, 11}\left(x, b^{\prime}\right) X_{\gamma, 11}\left(x, b^{\prime \prime}\right) d b^{\prime \prime} d b^{\prime}
\end{gathered}
$$

$I_{1}$ and $I_{2}$ are computed by numerical quadrature of (A15) and (A16), respectively. $\operatorname{SD}\left[S_{11}\right]$ and $\mathrm{CV}\left[S_{11}\right]$ for $\beta=0.75$ and 1.75 are shown in Figures $5 \mathrm{~b}$ and $5 \mathrm{c}$ and $6 \mathrm{~b}$ and $6 \mathrm{c}$, respectively.

Acknowledgments. The authors are indebted to Gedeon Dagan for stimulating discussions and suggestions. This research has been funded by the project MURST $40 \%$ "Trasporto di inquinanti nei corpi idrici naturali."

\section{References}

Ababou, R. D. B., D. McLauglin, L. W. Gelhar, and A. F. B. Tompson, Numerical three-dimensional saturated flow in randomly heterogeneous porous media, Transp. Porous Media, 4, 549-565, 1989.

Anderson, M. P., Comment on "Universal scaling of hydraulic conductivities and dispersivities in geologic media" by S. P. Neuman, Water Resour. Res., 27(6), 1381-1382, 1991.

Bellin, A., and Y. Rubin, Hydrogen: A spatially distributed random field generator for correlated properties, Stochastic Hydrol. Hydraul., in press, 1996.

Bellin, A., P. Salandin, and A. Rinaldo, Simulation of dispersion in heterogeneous porous formations: Statistics, first-order theories, convergence of computations, Water Resour. Res., 28(9), 2211-2227, 1992.

Bellin, A., Y. Rubin, and A. Rinaldo, Eulerian-Lagrangian approach for modeling of flow and transport in heterogeneous geological formations, Water Resour. Res., 30(11), 2913-2924, 1994.

Boggs, J. M., S. T. Young, L. M. Beard, L. W. Gelhar, K. R. Rehfeldt, and E. E. Adams, Field study in a heterogeneous aquifer, 1, Overview and site description, Water Resour. Res., 28(12), 3281-3291, 1992.

Cordes, C., and M. Putti, Finite element approximation of the operator on tetrahedra, SLAM J. Sci. Comput., in press, 1996.

Dagan, G., Solute transport in heterogeneous porous formations, $J$. Fluid Mech., 145, 151-177, 1984. 
Dagan, G., Flow and Transport in Porous Formations, Springer-Verlag, New York, 1989.

Dagan, G., Transport in heterogeneous porous formations: Spatial moments, ergodicity, and effective dispersion, Water Resour. Res., 26(6), 1281-1290, 1990.

Dagan, G., Dispersion of passive solute in non-ergodic transport by steady velocity fields in heterogeneous formations, J. Fluid Mech., 223, 197-210, 1991.

Dagan, G., The significance of heterogeneity of evolving scales and of anomalous diffusion to transport in porous formations, Water Resour. Res., 30(12), 3327-3336, 1994.

Desbarats, A. J., and S. Bachu, Geostatistical analysis of aquifer heterogeneity from the core scale to the basin scale: A case study, Water Resour. Res., 30(3), 673-684, 1994.

Feder, J., Fractals, Plenum, New York, 1988.

Fiori, A., Finite-Peclet extension of Dagan's solutions to transport in anisotropic heterogeneous formations, Water Resour. Res., 32(1), 193-198, 1996.

Fisher, H. B., E. J. List, R. C. Y. Koh, J. Imberger, and N. H. Brooks, Mixing in Inland and Coastal Waters, Academic, San Diego, Calif., 1979.

Forsyth, P. A., A control volume finite element approach to NAPL groundwater contamination, SIAM J. Sci. Comput., 12(5), 10291057, 1991.

Furtado, F., J. Glimm, W. B. Lindquist, and F. P. Pereira, Multi-length scale calculations of mixing length growth in tracer floods, paper presented at Emerging Technologies Conference, Inst. for Improved Oil Recovery, Houston, Tex., 1990.

Furtado, F., J. Glimm, W. B. Lindquist, F. P. Pereira, and Q. Zhang, Time dependent anomalous diffusion for flow in multi-fractal porous media, in Proceedings of the Workshop on Numerical Methods for the Simulation of the Multiphase and Complex Flow; Lect. Notes Phys., vol. 1, edited by T. M. M. Verheggan, pp. 79-89, Springer-Verlag, New York, 1991.

Gelhar, L. W., Stochastic subsurface hydrology from theory to applications, Water Resour. Res., 22(9), 135S-145S, 1986.

Gelhar, L. W., C. Welty, and K. R. Rehfeldt, A critical review of data on field-scale dispersion in aquifers, Water Resour. Res., 28(7), 1955$1974,1992$.

Gelhar, L. W., C. Welty, and K. R. Rehfeldt, Reply, Water Resour. Res., 29(6), 1867-1869, 1993

Glimm, J., and D. H. Sharp, A random field model for anomalous diffusion in heterogeneous porous media, J. Stat. Phys., 62, 415-424, 1991.

Glimm, J., W. B. Lindquist, F. P. Pereira, and R. Peierls, The multifractal hypothesis and anomalous diffusion, Rev. Bras. Mat. Aplic. Comput., 11, 189-207, 1992.

Glimm, J., W. B. Lindquist, F. P. Pereira, and Q. Zhang, A theory of macrodispersion for the scale-up problem, Transp. Porous Media, 13, 97-122, 1993

Gradshteyn, I. S., and I. M. Ryzhik, Table of Integrals, Series, and Products, Academic, San Diego, Calif., 1980.

Hess, K. M., S. H. Wolf, and M. A. Celia, Large-scale natural gradient tracer test in sand and gravel, Cape Cod, Massachusetts, 3, Hydraulic conductivity variability and calculated macrodispersivities, Water Resour. Res., 28(8), 2011-2017, 1992.

Kemblowski, M. W., and J. C. Wen, Contaminant spreading in stratified soils with fractal permeability distribution, Water Resour. Res., 29(2), 419-425, 1993.

Kitanidis, P. K., Prediction by the method of moments of transport in heterogeneous formation, J. Hydrol., 102, 453-473, 1988.

Lake, L. W., Enhanced Oil Recovery, Prentice-Hall, Englewood Cliffs, N. J., 1989.

Lavallèe, D., S. Lovejoy, D. Schertzer, and P. Ladoy, Nonlinear vari- ability and landscape topography: Analysis and simulation, in Fractals in Geography, edited by L. D. Cola and N. Lam, pp. 158-192, Prentice-Hall, Englewood Cliffs, N. J., 1993.

Mandelbrot, B. B., The Fractal Geometry of Nature, W. H. Freeman, New York, 1983.

Neuman, S. P., Universal scaling of hydraulic conductivities and dispersivities in geologic media, Water Resour. Res., 26(8), 1749-1758, 1990.

Neuman, S. P., Reply, Water Resour. Res., 27(6), 1383-1384, 1991

Neuman, S. P., Comment on "A critical review of data on field-scale dispersion in aquifers" by L. W. Gelhar, C. Welty, and K. R. Rehfeldt, Water Resour. Res., 29(6), 1863-1865, 1993.

Neuman, S. P., Generalized scaling of permeabilities: Validation and effect of support scale, Geophys. Res. Lett., 21(5), 349-352, 1994.

Neuman, S. P., On advective transport in fractal permeability and velocity fields, Water Resour. Res., 31(6), 1455-1460, 1995.

Osnes, H., Stochastic analysis of head spatial variability in bounded rectangular heterogeneous aquifers, Water Resour. Res., 31(12), 2981-2990, 1995.

Pickens, J. F., and G. E. Grisak, Scale-dependent dispersion in a stratified granular aquifer, Water Resour. Res., 17(4), 1191-1211, 1981.

Rajaram, H., and L. W. Gelhar, Plume scale-dependent dispersion in heterogeneous aquifers, 2, Eulerian analysis and three-dimensional aquifers, Water Resour. Res., 29(9), 3261-3276, 1993.

Rajaram, H., and L. W. Gelhar, Plume-scale dependent dispersion in aquifers with a wide range of scales of heterogeneity, Water Resour Res., 31(10), 2469-2482, 1995.

Rubin, Y., Flow and transport in bimodal heterogeneous formations, Water Resour. Res., 31(10), 2461-2468, 1995.

Rubin, Y., and G. Dagan, Stochastic analysis of boundary effects on head spatial variability in heterogeneous aquifers, 1 , Constant head boundary, Water Resour. Res., 24(10), 1689-1697, 1988.

Rubin, Y., and G. Dagan, Stochastic analysis of boundary effects on head spatial variability in heterogeneous aquifers, 2, Impervious boundary, Water Resour. Res., 25(4), 707-712, 1989.

Sposito, G., W. A. Jury, and V. K. Gupta, Fundamental problems in the stochastic convection-dispersion model of solute transport in aquifers and field soils, Water Resour. Res., 22(1), 77-88, 1986.

Sudicky, E. A., A natural gradient experiment on solute transport in a sand aquifer: Spatial variability of hydraulic conductivity and its role in the dispersion process, Water Resour. Res., 22(13), 2069-2082, 1986.

Taylor, G. I., Diffusion by continuous movements, Proc. London Math. Soc., 20(2), 196-212, 1921.

Zhang, Q., A multi-length scale theory of the anomalous mixing length growth for tracer flow in heterogeneous porous media, J. Stat. Phys., 66, 485-501, 1992.

A. Bellin, Dipartimento di Ingegneria Civile e Ambientale, Università di Trento, via Mesiano 77, Trento, I-38050, Italy. (e-mail: alberto.bellin@ing.unitn.it)

A. Fiori, Dipartimento di Scienze dell'Ingegneria Civile, Terza Università di Roma via C. Segre 60, Rome I-00146, Italy. (e-mail afiori@cidra.ing.uniromal.it)

M. Pannone, Dipartimento di Ingegneria e Fisica dell'Ambiente Università della Basilicata via della tecnica 3, Potenza I-85100, Italy. (e-mail: marilena@idra.unipd.it)

A. Rinaldo, Istituto di Idraulica "G. Poleni” Università di Padova via Loredan 20, Padova, I-35131, Italy. (e-mail: rinaldo@idra.unipd.it)

(Received April 8, 1996; revised August 8, 1996; accepted August 12, 1996.) 